\title{
Association of IL-8-Inducing Strains of Diffusely Adherent Escherichia coli with Sporadic Diarrheal Patients with Less Than 5 Years of Age
}

\author{
Ismail Mustafa Meraz ${ }^{1}$, Kentaro Arikawa ${ }^{1}$, Hiromi Nakamura ${ }^{1,2}$, Jun Ogasawara ${ }^{2}$, Atsushi Hase ${ }^{2}$ and Yoshikazu Nishikawa \\ ${ }^{1}$ Department of Food and Human Health Sciences, Graduate School of Human Life Science, Osaka City University; ${ }^{2}$ Department of \\ Microbiology and Health, Osaka City Institute of Public Health and Environmental Sciences; Osaka, Japan
}

\begin{abstract}
The role of diffusely adherent Escherichia coli (DAEC) in diarrheal disease has been controversial. However, DAEC strains were recently implicated in diarrheal disease in developing countries. To clarify whether DAEC are prevalent among sporadic cases of diarrheal illness in Osaka City, Japan, E. coli strains isolated between July 1997 and March 2000 during diarrheagenic $E$. coli (DEC) investigation were retrospectively examined. DAEC strains were recognized among 41 (4.4\%) of 924 patients and formed the biggest subgroup of DEC. Previously, we reported that some DAEC strains caused epithelial cells to secrete as much IL-8 as enteroaggregative $E$. coli strains did. In this study, we attempted to evaluate epidemiologically whether the ability of DAEC to induce IL-8 was involved in the pathogenesis. Relationship among patient age, symptoms, Afa adhesins, season and IL-8 induction were examined. The subgroup of DAEC that possessed Afa genes and/or induced a high level of IL-8 was significantly prevalent among patients age 1 to 4 years; however total DAEC was not significantly high among the children compared to other age group. IL-8 inducing DAEC seems to play a role in causing sporadic diarrheal illnesses, particularly in pediatric fields. Investigations highlighting the relationship between IL-8 induction and enteropathogenicity are clearly necessary to confirm the role of DAEC in infectious enteritis.
\end{abstract}

Key-Words: DAEC, IL-8, Afa, age, epidemiology.

Escherichia coli is the predominant facultative anaerobe of the normal colon flora. However, particular clones can cause diarrheal disease. The diarrheagenic E. coli (DEC) are classified according to pathogenesis as enteropathogenic E. coli (EPEC), enterotoxigenic E. coli (ETEC), enteroinvasive E. coli (EIEC), enterohemorrhagic $E$. coli (EHEC), and enteroaggregative $E$. coli (EAEC) [1].

Diffusely adherent $E$. coli (DAEC) has recently been recognized as the sixth class of DEC [2,3]. DAEC are defined as E. coli that possess no typical markers for other categories of diarrheagenic E. coli and that uniformly adhere to the entire surface of HEp-2 cells or HeLa cells. However, etiological role of DAEC strains in diarrheal disease has been controversial [4-6]. DAEC are probably comprised of heterogeneous groups of organisms with variable virulence because of the definition; it is unlikely that the ability to adhere to HEp-2 cells per se ensures enteropathogenicity.

It was reported that some DAEC strains use afimbrial adhesive sheaths (Afa) as a colonization factor for intestinal tract infection $[4,7,8]$. Afa were originally found as the colonization antigens of uropathogenic E. coli [9]. The Dr adhesins recognize the Dr antigen, which consists of the exposed domain of the decay-accelerating factor [10]; Dr adhesins are encoded on the same gene clusters and are considered to be subtypes of Afa [11]. DAEC strains can be classified into two large groups of Afa/Dr and non-Afa/Dr DAEC before considering their etiological role.

Received on 11 August 2006; revised 8 January 2007.

Address for correspondence: Prof. Yoshikazu Nishikawa, Department of Food and Human Health Sciences, Graduate School of Human Life Science, Osaka City University, 3-3-138 Sugimoto, Sumiyoshi-ku, Osaka 558-8585, Japan. Phone: +81-6-6605-2910.. Fax: +81-6-6605-2910. E-mail: nisikawa@life.osaka-cu.ac.jp.

The Brazilian Journal of Infectious Diseases 2007;11(1):44-49. (C) 2007 by The Brazilian Journal of Infectious Diseases and Contexto Publishing. All rights reserved.
The ability to trigger the secretion of inflammatory cytokines from mucosal epithelial cells has been suggested as a virulence factor of pathogens. Diarrheagenic EAEC can cause a large amount of the proinflammatory chemokine IL-8 to be released from human intestinal epithelial cells. Fecal IL8 elevation was suggested to correlate with the severity of clinical symptoms [12-14]. Previously, we have found that some DAEC strains also could induce IL-8 as much as EAEC did $[15,16]$.

In this study, we reviewed retrospectively the prevalence of DAEC among E. coli strains isolated during the previous investigation [17]. Given the above-mentioned knowledge about putative virulent properties of DAEC, their etiological role is discussed in attempts to correlate patient age, symptoms, season, Afa/Dr adhesins, and the ability to induce IL- 8 because clinical microbiologists need criteria to predict the diarrheagenicity of isolated DAEC strains.

\section{Materials and Methods}

Bacteria

Stored strains isolated from 924 specimens during the investigation between July 1997 and March 2000 [17] were reexamined for DAEC. Adhesion tests to HEp-2 cells in culture were performed as described before [18]. The DAEC strains were assigned to subgroups according to the scheme described by Le Bouguénec et al. [11] and were examined for their IL-8 induction [15,16]. Twenty-three strains of EAST1EC, E. coli that does not possess any diarrheagenic characteristics except the EAEC heat-stable toxin 1 (EAST1) gene, had also been included as putative DEC in the investigation, because an outbreak due to EAST1EC O166:H15 occurred in Osaka City in 1996 [19, 20]. However, three strains (V255, V594, V915) that had been assigned to the EAST1EC in a previous report [17] were analyzed as DAEC in this retrospective study since 
their diffuse adhesion on HEp-2 cells was confirmed to be reproducible. Each strain of O86a (strain V827) and O146 (strain V422) had been identified as class II EPEC in previous report [17] because the strains did not react with primers for eae in PCR, but their O serogroups fit into a scheme of EPEC and their adhesion to HEp-2 cells suggested their enteropathogenicity [21]. In this report these two strains were also classified as DAEC since the organisms had shown typical diffuse adhesion.

\section{Pulsed Field Gel Electrophoresis}

PFGE was performed according to the method of Izumiya et al. [22].

\section{Statistics}

Chi-square for independence test was used for statistical analysis.

\section{Results}

A total of 41 strains of DAEC was identified (Table 1). DAEC strains were examined for their clonal similarity using PFGE, and all DAEC strains including those of the same serogroups showed patterns clearly distinguishable from each other (data not shown). Classical DEC strains had been detected from 29 samples (3.1\%, 29/924): 15 EPEC, 10 EHEC, and 4 ETEC [17]. EAEC and EAST1EC were isolated from other 13 and 20 samples, respectively. By contrast, the DAEC strains were the largest group of possible pathogens and accounted for $40 \%(41 / 103)$ of total DEC (Figure 1). DAEC was 1.3 times more prevalent than all typical DEC (EHEC, EPEC, and ETEC) among sporadic diarrheal patients in Osaka City. Recent epidemiological studies have convincingly implicated DAEC strains as a cause of diarrhea $[4,5,23,24]$. If all of the DAEC strains isolated in this study were causative agents, the morbidity of DAEC infection would be higher than the total of well-established DEC. If DAEC strains were added to DEC, the isolation rate of DEC $(11.1 \%, 103 / 924)$ would be 1.6 times higher than Salmonella (6.8\%, 63/924). However, because the pathogenicity and virulence factors of DAEC have not been fully clarified, we hesitated to regard all DAEC strains as an enteric pathogen.

The overall association of infection with bacterial pathogens and diarrhea in young children is well recognized. EPEC and EHEC were significantly prevalent among patients age 1 to 3 years (Table 2 ). It was expected that DAEC could be significantly prevalent among children if the organisms were diarrheagenic. However, in the present study, total DAEC was not significantly prevalent in the age group, although several groups have reported a positive association between DAEC and diarrhea when studied populations were age stratified [4,23-26].

We suppose that a subpopulation of DAEC with additional virulence properties could be diarrheagenic for children. To get clues concerning enteropathogenicity of DAEC, we attempted to find children-associated properties of the organisms and noticed that only the DAEC strains that had Afa/Dr genes and/ or induced high amount of IL-8 were significantly prevalent among patients age 1 to 4 years (Table 2). The present data suggest the importance of discerning virulence properties to diagnose diarrhea attributable to DAEC.

From the standpoint of IL-8 secretion, we searched the threshold that could produce significant difference between children and other age group. Strains that caused T84 cells to secrete more than 9,12 , or $14 \mathrm{ng} / \mathrm{mL}$ IL-8 were significantly prevalent among patients age 1 to 4 years. No significant difference was observed in the age-stratified distribution of strains inducing IL-8 less than $9 \mathrm{ng} / \mathrm{mL}$. IL-8-inducing ability could be a marker to distinguish diarrheagenic strains among DAEC strains; although, the threshold of IL-8 secretion to distinguish diarrheagenic DAEC was not established at present since number of strains were not enough and strains isolated from healthy persons were not included for the analysis.

The DAEC was composed of 19 strains of Afa/Dr group and 21 strains of non-Afa/Dr (Table 1). Germani et al. reported that afa-possessing DAEC strains isolated from 2- to 6-yearsold children were important [8]. Afa/Dr DAEC strains were found to be significantly prevalent among patients age 1 to 4 years (Table 2, $\mathrm{p}=0.033$ ), when compared to other age groups of patients in this study. In the investigation we could not obtain fecal samples from healthy person as controls. However, present analysis likely suggests that Afa/Dr DAEC is involved in diarrheal diseases among young children since EPEC, a representative enteric pathogen in pediatric fields, was also detected at significantly high frequency among young children (Table 2).

Although virulence traits of Afa/Dr DAEC strains were well described in an excellent review by Servin [2], factors contributing to IL-8 induction have not yet been clarified. Diffuse adhesion is presumably insufficient to cause pathological changes at intestinal sites, and some DAEC strains that have additional factors can cause epithelial cells to secrete the chemokine IL-8. In our recent report, we noted flagella as a potent stimulant of IL-8 induction, particularly in Afa/Dr DAEC strains [15]; invasion is also likely to play an important role in non-Afa/Dr DAEC strains in causing epithelial cells to secrete IL-8 [16].

The major clinical symptom of the patients carrying DAEC stains was loose or watery diarrhea (Table 1). Mucus and blood was found among nine patients with DAEC (22\%, 9/ 41). Vomiting was reported in only four cases in our study; although, a case-control study reported that DAEC was significantly associated with vomiting rather than diarrhea among children [27]. Clinical symptoms of patients from whom low inducer strains were isolated were not mild compared to those of the patients infected with high inducers. It is possible that low inducers, particularly non-Afa strains, were not the causing agents in those patients.

The cumulative isolation rate of each subgroup of DEC during this study was assessed by season (Figure 2). When EPEC, ETEC, and EHEC were treated as a group of well- 
Table 1. List of DAEC strains isolated through the investigation

\begin{tabular}{|c|c|c|c|c|c|c|c|c|c|}
\hline \multirow{2}{*}{ Age } & \multicolumn{5}{|c|}{ Symptoms } & \multirow{2}{*}{$\begin{array}{c}\text { Afa } \\
\text { subtype }\end{array}$} & \multirow{2}{*}{ Motility } & \multirow{2}{*}{$\begin{array}{l}\text { IL-8 on } \\
\text { T84b } \\
\text { (ng/ml) }\end{array}$} & \multirow{2}{*}{ Other agents } \\
\hline & Serotypes & Fever $\left({ }^{\circ} \mathrm{C}\right)$ & Cramps & Vomiting & Diarrhea $^{a}$ & & & & \\
\hline 4 & OUT: H18 & & $?$ & $?$ & $\mathrm{~W}$ & AfaE1 & + & 17.3 & \\
\hline 1 & $\mathrm{O} 1: \mathrm{H} 28$ & 39.5 & $?$ & $?$ & $\mathrm{~W}$ & AfaEX & + & 14.6 & \\
\hline 4 & OUT: H4 & & + & $?$ & $\mathrm{~W}$ & AfaEX & + & 14.6 & \\
\hline 30 & UT & & + & $?$ & $\mathrm{~L}$ & $?$ & + & 13.8 & \\
\hline 2 & $\mathrm{O} 1: \mathrm{H} 4$ & 38.3 & $?$ & $?$ & $\mathrm{~L}$ & AfaEX & + & 13.7 & \\
\hline 2 & UT & & $?$ & $?$ & $M \& B$ & $?$ & + & 12.3 & \\
\hline 1 & UT & & $?$ & $?$ & $M \& B$ & $?$ & + & 12 & \\
\hline 6 & O114: HUT & & + & $?$ & $\mathrm{~L}$ & $?$ & + & 11.1 & \\
\hline 46 & OUT: H4 & & $?$ & $?$ & $\mathrm{~L}$ & AfaEX & + & 10.7 & \\
\hline 3 & O86a: H18 & & + & $?$ & $M \& B$ & AfaEX & + & 9.8 & \\
\hline 1 & UT & & $?$ & $?$ & $\mathrm{~L}$ & $?$ & + & 9.3 & \\
\hline 2 & O116: HUT & 37.6 & $?$ & $?$ & Diarrhea & $?$ & + & 9 & \\
\hline 9 & UT & & + & $?$ & $\mathrm{~L}$ & $?$ & + & 8.9 & C. jejuni \\
\hline 3 & OUT: H5 & 39.1 & $?$ & + & $\mathrm{W}$ & AfaE1 & + & 8.8 & \\
\hline 12 & O8: HUT & & + & $?$ & $M \& B$ & $?$ & + & 8.8 & Salmonella $\mathrm{O} 8$ \\
\hline 50 & UT & & $?$ & $?$ & $\mathrm{~W}$ & $?$ & + & 8.5 & \\
\hline $2 \mathrm{mo}$ & OUT: H? & 38.4 & $?$ & $?$ & $\mathrm{~L}$ & $?$ & $?$ & 8 & \\
\hline $2 \mathrm{mo}$ & O28ac: H20 & + & + & $?$ & $\mathrm{~W}$ & $?$ & + & 7.5 & \\
\hline 9 & O143:H4 & 38.5 & + & + & $\mathrm{BD}$ & $\mathrm{Afa} / \mathrm{Dr}^{?}$ & $?$ & 7.3 & \\
\hline 11 & O18:H4 & & + & $?$ & $\mathrm{AP}$ & $?$ & + & 5.4 & \\
\hline
\end{tabular}

${ }^{\mathrm{a}} \mathrm{B}=$ =blood was contained; $\mathrm{L}=$ Loose stool; $\mathrm{M}=$ Mucus; $\mathrm{W}=$ Watery; $\mathrm{BD}=\mathrm{Bloody}$ diarrhea; $\mathrm{AP}=\mathrm{Abdominal}$ pain. bMean of three to four tests. The data are based on our previous reports $[15,16]$. 
Table 2. Prevalence of each subpopulation of diarrheagenic E. coli in different age groups ${ }^{a}$

\begin{tabular}{|c|c|c|c|c|c|c|}
\hline \multirow[t]{2}{*}{ Pathogens } & \multicolumn{4}{|c|}{$\mathrm{N}(\%)$ of infected patients } & \multirow[b]{2}{*}{ Adults } & \multirow[b]{2}{*}{ Total } \\
\hline & $<1$ year & $1-4$ years & $5-12$ years & 13-19years & & \\
\hline EPEC & $1(0.9)$ & $9(2.3)^{\mathrm{b}}$ & $4(1.5)$ & $1(2.7)$ & 0 & 15 (1.6) \\
\hline EHEC & 0 & $6(1.5)$ & $2(0.8)$ & $1(2.7)$ & $1(0.8)$ & $10(1.1)$ \\
\hline ETEC & 0 & 0 & 0 & 0 & $4(3.2)$ & $4(0.4)$ \\
\hline EPEC+EHEC & $1(0.9)$ & $15(3.9)^{c}$ & $6(2.3)$ & $2(5.5)$ & $1(0.8)$ & $25(2.7)$ \\
\hline EAEC & $1(0.9)$ & 5 (1.3) & 3 (1.1) & 0 & $4(3.2)$ & 13 (1.4) \\
\hline EAST1EC & $2(1.8)$ & $8(2.1)$ & $2(0.8)$ & $2(5.5)$ & $6(4.8)$ & $20(2.2)$ \\
\hline DAEC & 4 (3.5) & $19(4.9)$ & $11(4.3)$ & 0 & $6(4.8)$ & $40(4.3)$ \\
\hline $\mathrm{Afa} / \mathrm{Dr}$ & 0 & $\begin{array}{c}12(3.1) \\
(\mathrm{P}=0.033)\end{array}$ & $4(1.5)$ & 0 & $2(1.6)$ & $18(2.0)$ \\
\hline Non-Afa/Dr & $4(3.5)$ & $7(1.8)$ & $6(2.3)$ & 0 & $4(3.2)$ & $21(2.3)$ \\
\hline High IL-8 Inducer ( $\geq 14$ ng/mL) ${ }^{d}$ & 0 & $\begin{array}{c}3(0.8) \\
(\mathrm{P}=0.04)\end{array}$ & 0 & 0 & 0 & $3(0.3)$ \\
\hline High IL-8 Inducer ( $\geq 12$ ng/mL) ${ }^{d}$ & 0 & $\begin{array}{c}6(1.5) \\
(\mathrm{P}=0.02)\end{array}$ & 0 & 0 & $1(0.8)$ & $7(0.76)$ \\
\hline High IL-8 Inducer ( $\geq 11$ ng/mL) ${ }^{d}$ & 0 & $\begin{array}{c}7(1.8) \\
(\mathrm{P}=0.06)\end{array}$ & 0 & 0 & $1(0.8)$ & $8(0.9)$ \\
\hline High IL-8 Inducer ( $\geq 9$ ng/mL) ${ }^{d}$ & 0 & $\begin{array}{c}9(2.3) \\
(\mathrm{P}=0.018)\end{array}$ & $1(0.4)$ & 0 & $2(1.6)$ & $12(1.3)$ \\
\hline Afa/Dr High Inducer & 0 & $\begin{array}{c}5(1.3) \\
(\mathrm{P}=0.04)\end{array}$ & 0 & 0 & 1 & 6 \\
\hline Non-Afa/Dr High Inducer & 0 & $4(1.0)$ & 1 & 0 & 1 & 6 \\
\hline Number of patients & 114 & 387 & 256 & 36 & 126 & 919 \\
\hline
\end{tabular}

Figure 1. Proportion of each group of diarrheagenic E. coli (DEC).

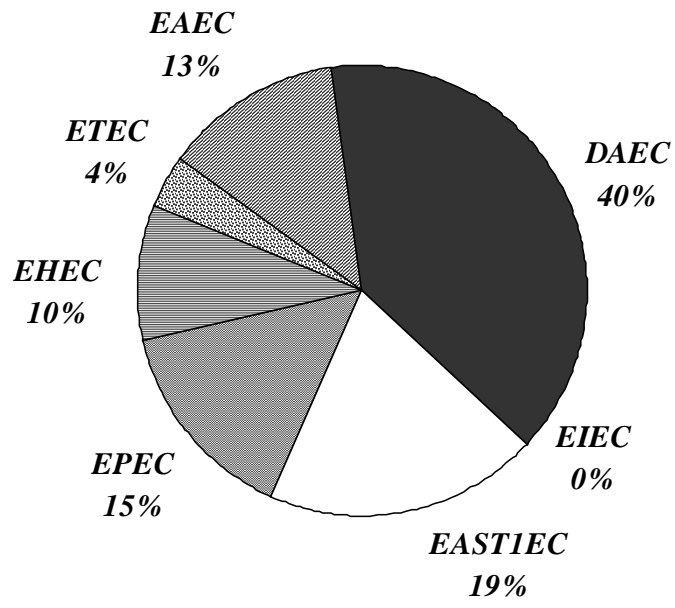

Figure 2. Seasonal distribution of DAEC along with EAEC, EAST1EC, and EPEC plus EHEC. Here Mar to May was considered as Spring, Jun to Aug was Summer, Sept to Nov was Autumn and Dec to Feb was Winter. The isolation index is expressed as a percentage of the number of isolates per number of samples during each season. The isolation rate $(4.3 \%, 16 /$ 374) of EHEC and EPEC in the summer season was significantly high ( $\mathrm{p}=0.015)$ compared to the other season $(1.6 \%, 9 / 550)$. Although four ETEC patients were included for analysis of the seasonal distribution in the text, ETEC were excluded in this graph since they were all imported cases.

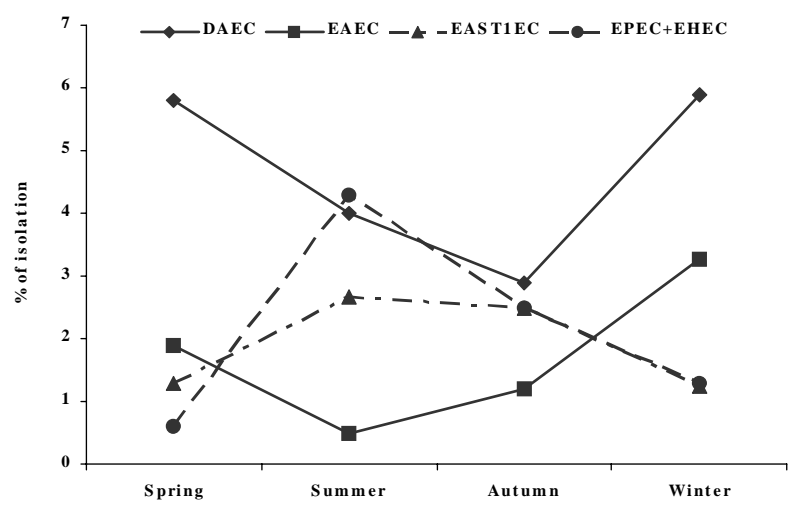


established DEC, the isolation rate of these typical DEC in the summer season $(5.1 \%, 19 / 374)$ was clearly high compared to the other season $(1.8 \%, 10 / 550)(\mathrm{p}=0.005)$. On the other hand, total DAEC were likely prevalent from winter $(5.9 \%, 9 / 153)$ to spring $(5.8 \%, 9 / 155)$ compared to autumn $(2.9 \%, 7 / 242)$ and summer $(4.3 \%, 16 / 374)$. This finding may suggest that most cases due to DAEC were not foodborne. Some DAEC strains may infect opportunistically damaged intestinal epithelia since viral diarrheal disease tends to increase during the cooler season in the pediatric population. However, as far as the highly inductive strains which caused IL-8 secretion more than $9 \mathrm{ng} /$ $\mathrm{mL}$ were concerned, they seem to be frequent in summer $(1.6 \%$, $6 / 374)$ compared to spring $(0.6 \%, 1 / 155)$, autumn $(1.2 \%, 3 / 242)$, or winter $(1.3 \%, 2 / 153)$. Although no significant difference was observed, the finding lets us expect that the subpopulation of DAEC might cause foodborne infection in summer.

\section{Conclusion}

In conclusion, DAEC is the most prevalent fecal isolate among DEC. Some DAEC strains that can cause epithelial cells to secrete high amount of chemokine IL-8 or that possess Afa/Dr gene are likely diarrheagenic particularly in pediatric field. Epidemiological investigations highlighting the relationship between these properties and diarrheagenicity could confirm the role of DAEC in infectious enteritis. The characteristics of DAEC strains isolated from healthy persons and those from diarrheal specimens should be compared in future studies.

\section{Acknowledgements}

Eleven hospitals were selected as sentinels in order to cover the Osaka City area for this investigation. We thank the following participants for their contribution to this project: Drs. Hidenori Imaishi, Akira Fukumoto, and Akihiko Hori at NTT West Osaka Hospital; Drs. Isao Yamamori, Kenji Suzuki, and Shigeko Saitoh at Nagayoshi General Hospital; Drs. Tetsuji Goto, Hideki Yoshida, Hironari Niimi, Eiji Ikeda, and Yoshihiro Sakaue at Osaka City General Medical Center; Drs. Atsushi Ono and Tetsuro Yoshida at Saiseikai-Izuo Hospital; Drs. Ryouji Ebina and Katsushi Kaji at Nakano Children Hospital; Drs. Gen Unishi, Mayumi Ideno, Miyoko Tsuji, Toshiaki Hayashi, and Hiroaki Sudo at Saiseikai-Noe Hospital; Drs. Yuriko Tsubakio, Chiyo Iwamura, and Akitomo Yoshimura at Osaka City Juso Hospital; Drs. Yoshiro Morikawa and Miyoshi Kitazato at Yodogawa Christian Hospital; Dr. Hiroyuki Ichiba at Sumiyoshi Citizen's Hospital; Dr. Tomoyuki Kawamura at University Hospital, Osaka City University; Drs. Takeshi Goto and Michiko Suzuki at Kita Citizen’s Hospital.

\section{References}

1. Nataro J.P., Kaper J.B. Diarrheagenic Escherichia coli. Clin Microbiol Rev 1998;11:142-201.

2. Servin A.L. Pathogenesis of Afa/Dr diffusely adhering Escherichia coli. Clin Microbiol Rev 2005;18:264-92.

3. Kaper J.B., Nataro J.P., Mobley H.L. Pathogenic Escherichia coli. Nat Rev Microbiol 2004;2:123-40.
4. Giron J.A., Jones T., Millan Velasco F., et al. Diffuse-adhering Escherichia coli (DAEC) as a putative cause of diarrhea in Mayan children in Mexico. J Infect Dis 1991;163:507-13.

5. Jallat C., Livrelli V., Darfeuille Michaud A., et al. Escherichia coli strains involved in diarrhea in France: high prevalence and heterogeneity of diffusely adhering strains. J Clin Microbiol 1993;31:2031-7.

6. Echeverria P., Serichantalerg O., Changchawalit S., et al. Tissue culture-adherent Escherichia coli in infantile diarrhea. J Infect Dis 1992; 165:141-3.

7. Le Bouguénec C., Garcia M.I., Ouin V., et al. Characterization of plasmid-borne afa-3 gene clusters encoding afimbrial adhesins expressed by Escherichia coli strains associated with intestinal or urinary tract infections. Infect Immun 1993;61:5106-14.

8. Germani Y., Begaud E., Duval P., Le Bouguénec C. Prevalence of enteropathogenic, enteroaggregative, and diffusely adherent Escherichia coli among isolates from children with diarrhea in New Caledonia. J Infect Dis 1996;174:1124-6.

9. Labigne Roussel A.F., Lark D., Schoolnik G., Falkow S. Cloning and expression of an afimbrial adhesin (AFA-I) responsible for $\mathrm{P}$ blood group-independent, mannose-resistant hemagglutination from a pyelonephritic Escherichia coli strain. Infect Immun 1984;46:251-9.

10. Telen M.J., Hall S.E., Green A.M., et al. Identification of human erythrocyte blood group antigens on decay-accelerating factor (DAF) and an erythrocyte phenotype negative for DAF. J Exp Med 1988;167:1993-8.

11. Le Bouguénec C., Lalioui L., Du Merle L., et al. Characterization of AfaE adhesins produced by extraintestinal and intestinal human Escherichia coli isolates: PCR assays for detection of afa adhesins that do or do not recognize Dr blood group antigens. J Clin Microbiol 2001;39:1738-45.

12. Steiner T.S., Lima A.A., Nataro J.P., Guerrant R.L. Enteroaggregative Escherichia coli produce intestinal inflammation and growth impairment and cause interleukin-8 release from intestinal epithelial cells. J Infect Dis 1998;177:88-96.

13. Jiang Z.D., Greenberg D., Nataro J.P., et al. Rate of occurrence and pathogenic effect of enteroaggregative Escherichia coli virulence factors in international travelers. J Clin Microbiol 2002;40:4185-90.

14. Steiner T.S., Nataro J.P., Poteet Smith C.E., et al. Enteroaggregative Escherichia coli expresses a novel flagellin that causes IL-8 release from intestinal epithelial cells. J Clin Invest 2000;105:1769-77.

15. Arikawa K., Meraz I.M., Nishikawa Y., et al. Interleukin-8 secretion by epithelial cells infected with diffusely adherent Escherichia coli possessing Afa adhesin-coding genes. Microbiol Immunol 2005;49:493-503.

16. Meraz I.M., Arikawa K., Ogasawara J., et al. Epithelial cells secrete interleukin-8 in response to adhesion and invasion of diffusely adhering Escherichia coli lacking Afa/Dr genes. Microbiol Immunol 2006;50:159-69.

17. Nishikawa Y., Zhou Z., Hase A., et al. Diarrheagenic Escherichia coli isolated from stools of sporadic cases of diarrheal illness in Osaka City, Japan between 1997 and 2000: prevalence of enteroaggregative $E$. coli heat-stable enterotoxin 1 genepossessing E. coli. Jpn J Infect Dis 2002;55:183-90.

18. Nishikawa Y., Scotland S.M., Smith H.R., et al. Catabolite repression of the adhesion of Vero cytotoxin-producing Escherichia coli of serogroups 0157 and O111. Microb Pathog 1995; $18: 223-9$.

19. Nishikawa Y., Ogasawara J., Helander A., Haruki K. An outbreak of gastroenteritis in Japan due to Escherichia coli O166. Emerg Infect Dis 1999;5:300.

20. Zhou Z., Ogasawara J., Nishikawa Y., et al. An outbreak of gastroenteritis in Osaka, Japan due to Escherichia coli serogroup O166 : H15 that had a coding gene for enteroaggregative E-coli heat-stable enterotoxin 1 (EAST1). Epidemiol Infect 2002;128:363-71. 
21. Levine M.M., Nataro J.P., Karch H., et al. The diarrheal response of humans to some classic serotypes of enteropathogenic Escherichia coli is dependent on a plasmid encoding an enteroadhesiveness factor. J Infect Dis 1985; $152: 550-9$.

22. Izumiya H., Terajima J., Wada A., et al. Molecular typing of enterohemorrhagic Escherichia coli O157:H7 isolates in Japan by using pulsed-field gel electrophoresis. J Clin Microbiol 1997;35:1675-80.

23. Gunzburg S.T., Chang B.J., Elliott S.J., et al. Diffuse and enteroaggregative patterns of adherence of enteric Escherichia coli isolated from aboriginal children from the Kimberley region of Western Australia. J Infect Dis 1993; $167: 755-8$
24. Levine M.M., Ferreccio C., Prado V., et al. Epidemiologic studies of Escherichia coli diarrheal infections in a low socioeconomic level peri-urban community in Santiago, Chile. Am J Epidemiol 1993;138:849-69.

25. Scaletsky I.C., Fabbricotti S.H., Carvalho R.L.B., et al. Diffusely adherent Escherichia coli as a cause of acute diarrhea in young children in northeast Brazil: a case-control study. J Clin Microbiol 2002; 40 :645-8.

26. Baqui A.H., Sack R.B., Black R.E., et al. Enteropathogens associated with acute and persistent diarrhea in Bangladeshi children less than 5 years of age. J Infect Dis 1992;166:792-6.

27. Poitrineau P., Forestier C., Meyer M., et al. Retrospective case-control study of diffusely adhering Escherichia coli and clinical features in children with diarrhea. J Clin Microbiol 1995;33:1961-2. 